\title{
Reaching Challenging Population in TB Program Through Religious, Informal Leaders and Traditional Music in West Sumatera Province, Indonesia
}

\author{
Rizanda Machmud ${ }^{1}$, Fatmasari Purba ${ }^{2}$, Finny Fitry Yani Masrul ${ }^{3}$, Delyuzar ${ }^{4}$, Mekkla \\ Thompson $^{5}$, Artha Camellia ${ }^{6}$ \\ \{rizanda_machmud@yahoo.com ${ }^{1}$ \} \\ Community Medicine and Public Health Department, Universitas Andalas, Padang, Indonesia
}

\begin{abstract}
TB is a leading public health concern in Indonesia which ranks second on the list of high burden TB countries[1]. In West Sumatra 47\% of TB cases are undetected, diagnosed late, or received incomplete treatment because of low-level awareness and knowledge especially among the hardest to reach populations[2]. Community empowerment against tuberculosis project funded by USAID and lead by Networking For Community Welfare \& Health was implemented in West Sumatra, Indonesia, focuses on identify the best communication channel to reach those who lived vulnerable and remote areas. (1) Identify the best channel for hard to reach group by focus group discussion and in-depth interviews, brainstorming with the community (2) find solutions to solve the problem together, plan of action (3) use results to form focus groups to implement, recruit and train religious and informal leaders. Afterwards, the leaders conducted community outreach to increase community awareness and knowledge about TB and improve community health-seeking behaviour. Our strategies included empowerment, capacity building, and socialization TB through Traditional Music Media by conducted edutainment with free traditional music and ethnic theatricals, integrating information regarding TB signs and symptoms and the impact of non-treatment of cases and transmission to the music, and (4) conducted focus group discussion and depth interviews for evaluation the activities. The involvements of religious and informal leaders and the use of traditional music as communication channel improves the population's awareness of TB symptoms and access to TB testing and treatment, as well as reduce TB-related stigma. We observed an increase in the case detection rate from $47 \%$ to $85 \%$ during the study period. Religious, informal leaders and traditional music were the best communication channel to reach the vulnerable population especially those who lived in remote areas.
\end{abstract}

Keywords: Hard to Reach Population, Health communication, Tuberculosis, Stigma.

\section{Introduction}

Tuberculosis (TB) infection remains one of the biggest health problems in Indonesia, which ranked at second place on the list of global TB burden countries[2]. The government in many low- and middle-income countries, where the TB burdens are high, and continues to struggle to contain the spread of TB[1]. The Case Detection Rates (CDRs) in the Indonesian National Tuberculosis Program (NTP) recently have remained steady or slightly declined due to undernotification and barriers in access to diagnosis facilities[2]. 
TB control problems are associated with a low level of awareness and knowledge of TB, mainly due to the high level of TB stigma in the community[3, 4]. The stigma of TB in Indonesia is mainly related to the belief that TB as a hereditary disease, TB as a magic-related disease or $\mathrm{TB}$ as the disease of the indigent people, and it is shameful to have a family member with TB[2]. The stigma of infectious diseases, including the stigma of TB, is usually observed among isolated communities, a population with low education and low socioeconomic status, many of whom are not reached by the health care system [5]-[7]. Other hard to reached population include those living in urban slums, post-disaster area, remote areas, the poor, contacts of TB patients, people living with HIV/AIDS, and malnourished people, among whom TB is usually under-detected[8]-[11].

As the largest archipelago country in the world, Indonesia's area consists of $80 \%$ of water, and only $20 \%$ of land covering an area of 2 million $\mathrm{km} 2$. The majority of the 235 million Indonesian population are spread over in five main islands, namely Sumatra, Java, Kalimantan, Sulawesi, and Irian Jaya, and the remaining live in about 13,000 smaller islands, mainly of which are isolated. We conducted this study in the West Sumatera Province, more specifically in the District of Solok and Mentawai Island. These two areas are located in the western part of Sumatera Island, which is quite remote in the earthquake-prone area. Both area, which was hit by multiple earthquakes in the last decades, have lower Human Development Index compared to other regions in the West Sumatera province. The TB coverage detection rate is also the lowest than compared to those in the other districts[2]. Intensified efforts are required to reduce TB transmission and accelerate reductions in $\mathrm{TB}$ incidence, particularly in those difficult terrains or hard to reach populations [8], [10], [11].

In order to effectively reach target populations, public health promotion efforts have tried to engage faith-based organisations over the past few years [12], [13]. The involvement of religious leaders in health-related interventions has generally been found to improve the participation of their congregations in these interventions and thus promote positive health outcomes [13]-[15]. Religious and spiritual leaders are some of the most credible people who influence the thoughts, emotions, and actions of a person of faith [15]. People of faith often make a conscious decision to behave in a manner that is consistent with the expectations of their religious or spiritual messages [14], [16].

Researches in another setting have shown the need to address cultural health beliefs about the locus of control in the design of developing programs TB care and treatment [14], [17]. The results of that study revealed that foreign-born Vietnamese clients tended to operate with an internal locus of control in health beliefs that attributed the resolution of health problems such as tuberculosis to chance rather than something that could be controlled by others [17].

People in West Sumatera are mostly Minang tribe, they hold culturally rooted health beliefs and practices. There is a folk tradition of the Minangkabau ethnic group which incorporates music, singing, story-telling called Saluang. It is very popular and usually performed for traditional ceremonies and festivals. Stories are delivered by both the acting and the singing and are mostly based on Minangkabau legends and folktales. Many researchers report that the cultural approach can be a potential channel to deliver the edutainment of TB disease [17]. The aim of the study that we will focus and try to figure out what is the best channel that specific for West Sumatera characteristic, on identifying possible TB cases in vulnerable populations to find them, especially people living in urban slums, remote islands, post-disaster areas, the poor and contacts of TB patients and people living with HIV/AIDS, MDR-TB and malnourished people. We will develop and expand community-based models to achieve universal access to TB testing and treatment 


\section{Materials and Methods}

\subsection{Study setting}

This study is part of a larger study, the community empowerment against tuberculosis (CEPAT) project, which was funded by USAID and lead by Jaringan Kesehatan/Kesejahteraan Masyarakat $(J K M)$ or Networking For Community Welfare \& Health in West Sumatera. We implemented the project in Mentawai and Solok Districts. These districts were selected as they were post-disaster and remote areas were TB case detection rates were found to be low compared with other districts. We built upon our team's experience, modify and utilise the tools that JKM had already developed for community mobilisation, TB patient support, and community health worker outreach. We had full support from the Provincial Health office and District Health office in Solok and Mentawai Districts for material and resources as well. We had Monitoring and Evaluation officers who were under the Chief of Party's direction would be responsible for working with all team members to collect, collate, and analyzed project performance monitoring data.

\subsection{Research design}

This study was designed as an operational research with three steps: step one (1) mapping the best channel for hard to reach group by focus group discussion and depth interviews, brainstorming with the community about their problems; and step two (2) found the solution to solve the problem together, made plan of action and implementation of the program, and after that, step three (3) we made evaluation and measurements of outcomes.

Step 1: Exploring character and brainstorming with the community.

We conducted a qualitative study by FGD and depth interviews in Mentawai and Solok Districts among 32 TB officers, religious and informal leaders, and traditional artist. In Mentawai district, there is three in-depth interviews with TB officers and religious leaders, and two FGDs with informal leaders, and cadres. Meanwhile, in Solok there are four in-depth interviews with TB Officers, traditional artist, and two FGDs with informal leaders, and cadres.

We asked open-ended questions about their perceptions on determining which methods were best used to increase knowledge and awareness of the community to support the TB programs, finding solutions to solve the TB problem together (how to increase awareness, knowledge and thus case-finding rate). The interviews were recorded and transcribed verbatim. The transcripts were thematically analyzed. The analysts coded the transcripts and reviewed, discussed, and refined the coding schemes until consensus was reached. Emerging concepts were assessed using the constant comparative method from grounded theory. The venue is held in the centre of the village, led and supported by the head of the village, in the community in Mentawai and Solok district. We developed and expanded community-based models to achieve universal access to TB testing and treatment.

\section{Step 2: Intervention content and change mechanism.}

Initial engagement was tailored which is appropriate for community outreach based on FGD and in-depth interview in the first step. This supported community engagement, seeking to achieve effective health communication where the community was moving to run the TB program. The community empowerment activities should be done independently and without any coercion from outsiders - the spirit of the local community as a bottom-up process. 
The frequency of group sessions implemented, there were 10 Advocacy, communication and social mobilization (ACSM) for TB control training in community case finding; 1 religious leader training, 1 traditional informal leader training, including ethnic leader and patron-client, and 8 the village community cadres training. The participant was 25 per village ( 8 villages), for 250 total of a person.

Facilitators' professional background of the training: Our team had substantial experience in the control of TB, MDR-TB, and TB-HIV. Our consortium members had working relationships in that the JKM Director also served as adjunct lecturer at University of Indonesia (UI), University of Sumatera Utara (USU), and University of Andalas (UNAND), Westat, TB experts from two Thai universities, Prince of Songkla and Mahidol. We worked together under a U.S. NIH TB research training grant, and USU and UI work together on TB programs.

Each region had a different approach to set the suitable channel for hard to reach the population in Mentawai and Solok districts. In the Mentawai region, we conducted a religious approach. We set the meeting with all 5 religious leader Protestant pastors who were selected via purposeful sampling. Semi-structured interviews were conducted with Protestant pastors. The researchers approached the pastors from various Protestant denominations and invited to participate in this research. One of the researchers contacted them to explain the procedures and answer any questions. Agreement to an appointment for an interview was considered informed consent. The participants were invited to venue which are provided by District Health Office of Mentawai, we interviewed them regarding numerous TB topics. The interviews lasted 60 minutes on average. Moreover, interview questions were added or adapted based on the analysis of previous interviews in order to understand and test emerging concepts. Inclusion and thus the interviewing of participants was continued until no new information was gleaned. After that, we conducted religious leader training, including simulated questions that might be asked by the people in their communities and provided them all with TB materials. Materials or tools the participant used during and outside the group session, we trained the participant to include simulated questions that might be asked by the people in their communities and provided them with all TB materials.

In Solok region, we conducted cultural approach: performed a story-telling and singing of Saluang about TB, identify famous singer to sing about TB, where traditional music included 2 edutainment session with free traditional music and ethnic theatricals by the singer. We delivered through free traditional music and ethnic theatricals Saluang.

In the implementation process for traditional music, we approached the community formal and informal leader for permission to conduct the Saluang in their community in Solok District. We identified the famous singer in the village through the informal leader. We provided the information about TB to the singer. The singer composed the song to include TB information at which they will talk about TB to improve awareness of signs and symptoms of $\mathrm{TB}$ and knowledge about TB available services and to encourage health-seeking behaviour.

\section{Step 3: Evaluation and measurements of outcomes}

Under the Chief of Party's direction, the monitoring and evaluation (M\&E) officers were responsible for working with all team members to collect, collate, and analyzed project performance monitoring data. The M\&E officers developed the data collection forms and trained project staff and the community cadres to use the forms to collect and reported the data.

They train community cadres to randomly select attendees of traditional music performances or after religious preach where TB is discussed. The cadres asked them 5 questions about TB and record results on the activity log. Results were maintained in Excel spreadsheets that were discussed by the team during regular team planning meetings. Frequent 
discussion of performance allowed the team to increase or redirect efforts in a timely way to improve performance.

Ethical clearance. Ethical clearance was given by the committee ethics of the Faculty of Medicine at the Andalas University, Indonesia. Verbal informed consent from the participants was audio recorded. We kept anonymity during data analysis and presentation.

\section{Result}

The theme of reaching hard to reach the population in TB Program showed there were specific health communication channels which were appropriate for community outreach assisted in increase TB knowledge and Awareness for TB Program especially those who lived in the remote areas. Our findings had suggested areas of intersection between a patient's health care and their cultural-religious beliefs that need unique health communication channel in hard to reach population. This theme reflected there were a problem in the TB control program and the need of channel for hard to reach population. The derivation of themes: some examples of questions, meaning units, codes, categories, and sub-themes can be seen in Table 1.

The problem in TB control program consisted of three categories: KAP and awareness in TB are low that impact to TB coverage, the system on TB control in the community has not been built optimally, the problem with hard to reach population. Channel to reach hard to reach population consisted of one category that was the need a different channel to reach hard to reach the population in the different region.

\subsection{Problem in TB control program}

\subsubsection{KAP and awareness in $\mathrm{TB}$ are low that impact to $\mathrm{TB}$ coverage}

Almost all informant of TB officers, informal leaders and cadres raised the perceived KAP and awareness in TB were low that impact to TB coverage. This was due to that TB is still stigmatized, and the knowledge about the disease and treatment remains low. The community's knowledge of TB points out that they still hold the stigma of TB. Some statement arose from cadres: What is believed in the community is that TB is a hereditary disease, it is shameful to have a family member with TB, TB is a disease of indigent people, and TB is a magic-related disease.

TB officer both in Mentawai and Solok district mentioned the Case Detection Rates (CDRs) in the National Tuberculosis Program (NTP) recently have remained steady or slightly declined due to under-notification and barriers in access to diagnosis facilities. Our informant, medical doctor in primary health care both in Mentawai and Solok district, also mentioned Tuberculosis was a disease with many elusive patients. It meant that most patients were asymptomatic or simply had mild symptoms that were not clinically apparent at any level. The health provider realized that only a small fraction of the problem was seen on its surface. Due to the diversity found in the clinical manifestations of TB, not all of the patients in the community were identified by health care providers. Generally, only patients with severe manifestations were likely to be identified by the health care provider, while those without apparent clinical manifestations were not. This condition made the case detection were lower than the target program. 


\subsubsection{The system on TB control in the community has not been built optimally}

The informal leader and the cadres raised perceived there was no comprehensive and coordinated approach to engaging the community in supporting TB services, including helping patients stay on treatment, proactively identifying possible TB cases, and advocating for better quality TB services. So on TB officers realized that community systems had limited capacity to increase public knowledge of TB, support improved TB diagnosis and treatment.

\subsubsection{Hard to reach the population}

TB officer both in Mentawai and Solok mentioned about geographic burden in their area: they had a wide and remote area to reach TB patient. Sometimes there were landslide and storm and made the area isolated.

The description of our study area: Mentawai district consists of a cluster of islands large and small, amounting to 99 islands surrounded by the Indian Ocean. The most of resident lived in 4 main islands such as Siberut, Sipora, North Pagai and South Pagai. All the transportation between islands used a boat. The topography of Solok district varies greatly between plains, valleys and rolling hills and mountain, with a height of 329 meters - 1458 meters above sea level. The TB officers and cadres mentioned that they had to go by foot more than two hours for community outreach due to no access road. Mentawai and Solok district, both areas had many people living in urban slums, remote islands, post-disaster areas, the poor, malnourished, and contacts of TB patients. These districts were selected as they were post-disaster areas were TB case detection rates were found to be low compared with other districts.

\subsection{Channel to reach hard to reach the population}

\subsubsection{Different channel to reach hard to reach the population in the different region}

Our study found that it needs a different health communication channel for the different region based on their culture and belief. The characteristics of Mentawai district was a different culture with other districts in West Sumatra Province. This area had a different language, even most of all the community had a different religion. The TB officer and medical doctor as health provider perceived to have a challenge in health communication due to more than one langue in the area. But we had potential health communication channel in Mentawai. The occasion that people are people coming mostly in Mentawai: the informal leaders mentioned that almost people in Mentawai every Saturday come to the church to pray. We developed an approach for community outreach in a remote area in Mentawai. We made it through existing channels in the community such as through religious leader. The religious leaders in Mentawai mentioned they were willing to participate in promoting TB message during their speech. We made some models collaboration with community leaders and religious leaders to mobilize the community and socialize TB Program.

Meanwhile, in Solok, it had different geography and characteristic from Mentawai. The informal leaders in Solok mentioned people come mostly if there was a party in the village, it performed Saluang as traditional music. They met and chatted each other in the Fiesta. We developed an approach for community outreach in the remote area. We made it through existing channels in the community such as through cultural approach. The traditional music mentioned they were willing to participate in promoting $\mathrm{TB}$ message during their performance. We modified the song which is delivered by singing and was mostly based upon Minangkabau 
legends and folktales that contain education, information and communications to reduce the TB stigma in the community.

\subsubsection{Implementation of reaching hard to reach population initiative}

We have conducted community meetings for traditional musicians and religious leader. The activity delivered message about $\mathrm{TB}$ signs and symptoms of TB, and available services to encourage health-seeking behaviour. These activities were implemented in sub-districts through 208 religious meetings, 6 traditional culture meetings, and 85 musical events that have attracted hard-to-reach people from 2013 until 2015.

\subsubsection{Religious approach}

We had trained the 5 religious leaders to include simulated questions that might be asked by the people in their communities and provided them with all TB materials. We had trained community volunteers and leaders using the ACSM Training Curriculum, we utilized the tools as in our previous projects. The All religious leaders of Mentawai fully accepted and willingness to be apart in support for TB control program in their region. The religious leader, when they give a speech in religious activity, weekly or monthly religious meeting in society, they bring Information, Education, Communication TB materials such as brochures and leaflets for a community group, which is hard to reach population. As a religious community, the people of West Sumatera province are respected for their religious and informal leader.

\subsubsection{Cultural approach}

We had several meetings to develop the media with the traditional musician. We had conducted edutainment (Education Entertainment) by holding free traditional music Saluang to insert the information of TB signs and symptoms and also the impact of non-treatment of TB cases and its transmission. We had modified both the story-telling and the singing delivered the stories. The songs in Saluang were mostly based upon Minangkabau legends and folktales. We inserted and combined with TB message that contains education, information and communications to reduce the TB stigma in the community.

We can increase the CDRs in Mentawai were $47.42 \%$, and after four years of implementation, it became $85 \%$ in 2015 . The success rate had also increased $60.98 \%$ in 2012 to $85 \%$ in 2015. Moreover, for CDRs Solok district were $47.54 \%$, and after four years of implementation, it became 69.52 in 2015 . The success rate had also increased $60.98 \%$ in 2012 to $89.36 \%$ in 2015 .

\section{Discussion}

\subsection{The impact of the interventions}

Our study had successfully educated communities in a remote area by religious and cultural initiative. They actively participated as a health communication channel for vulnerable community outreach. They delivered education in TB symptoms during religious community meetings, traditional culture meetings, and musical events that attract hard-to-reach people. 
The impact of the interventions in our project had shown that the involvements multiple health communication of religious, informal leaders and village community cadres were strengthening TB networking to stakeholders and community groups for TB control program[18]. The impact of these interventions has resulted in an increase of case detection rates (CDR) in our project area. Our intervention had the same impact with Pirkani GS, et al. their study on the role of religious leaders about tuberculosis had an impact on case detection rate in Balochistan, Pakistan[15]

Our approach in line with the recent Rio political declaration as a result of World Conference Social Determinant of Health announces that countries agreed to achieve social and health equity through action on social determinants of health and well-being by a comprehensive inter-sectoral approach. It might be solved through three main actions (3): a) Involvement of organized communities and all levels of government - local, provincial and national. b) Solutions often lie beyond the health sector and require the engagement of many different sectors of government and society. c) Local leaders and governments could and should play a key role in TB control in Indonesia

\subsection{The role of cultural and religious contexts in health communication}

Based on our study, the role of the religious leader had proven leverage impact on another disease besides TB. The same study approach such as in Uganda that called faith-based initiatives in which the involvement of religious leaders and organizations in HIV/AIDS prevention has had a major impact[16]. Minetti A, et al. found that addressing spiritual issues can make such a difference in an individual's experience of illness - and often in health outcomes as well. This study was talking about reaching hard-to-reach individuals in response vaccination for measles. Another study related to the role of religious contents in health communication was Toni-Uebari et al. His study on haemoglobinopathies found weaving spirituality into medical education has become a priority among integrative medicine leaders. It made sense that attention to spirituality is also important for health care providers themselves[15].

The cultural contexts also played a role in health communication. Establishing local culture such as Saluang with communities raising TB awareness among the general population. The traditional musicians talked about TB to improve awareness of signs and symptoms of $\mathrm{TB}$ and knowledge about TB available services and to encourage health-seeking behaviour. It could be both interesting communities because of events that were served were entertaining while still providing the benefits of TB knowledge to society.

A related study on the role of cultural content in health communication was from Houston $\mathrm{HR}$, et al. He found that development of a culturally sensitive educational intervention program could reduce the high incidence of tuberculosis among foreign-born Vietnamese. Furthermore, such efforts could play an important role in reducing the stigma associated with the disease.[17]

It is thought that community health through cultural approaches may have a greater impact on TB control. Given that health decision-making is often a communal rather than an individual process in many most low-income countries, including Indonesia, community health channel through cultural approaches seem warranted.

\subsection{The role of stigma in health promotion and disease control}

We identified gaps in knowledge about the cause of TB, inappropriate health care seeking behaviour and stigma towards TB. In other studies traditional beliefs such as "evil eye", Satan 
and witchcraft were the commonest perceived causes of TB[3]. In Tanzania, Pakistan just like in West Sumatera, has a significant number of people also mentioned that witcheraft could be the cause of TB[4]. These traditional beliefs might contribute to the spread of TB as most people with such beliefs may not visit health facilities.

\subsection{Lessons learnt from the intervention}

Discovering the broader context of a patient's life was critical to providing responsive care and assuring good in TB health outcomes[15]. It could increase awareness, knowledge, case finding rate, access to TB treatment, adherence and successful treatment.

Another lesson learns from our study that initial engagement should be tailored as health communication perceived to enhance message relevance and identify situations. We had to use tailoring message to distribute in the community outreach.

\subsection{The strengths and limitations of the study}

We had successfully educated communities by having trained staff or community leaders provide education in TB symptoms during religious community meetings, traditional culture meetings, and musical events that attract hard-to-reach people. We had empowered volunteers to increase case finding, trace TB contacts, and expand DOTS and provide them with checklists to ensure comprehensive services.

The strength of the study, in particular, due to local specific, our intervention project cannot implement in another region generally. It requires a review study before implemented. However, at least, if the characteristic of the community is similar, it can be applied to another region.

The limitation of the study, we are not enough exploring for all the component in cultural and religious contexts that play roles in health communication for TB. We assume there are still many things that can explore more.

\subsection{The implications for public health}

Health care providers may sometimes be uncomfortable talking about cultural-religious health beliefs and behaviours with patients and families[16]. It can say as a community health literacy problem; this condition becomes a challenge for TB officer in delivery TB promotion. The need for adequate language interpreters in health care settings is uniformly addressed, but it is also imperative for people to be able to communicate with leaders of their faith community[17]. These influential figures can help interpret what is happening on a spiritual level during a health crisis for patients and their families. Religious leaders can clarify which tenets cut across the branches of their faith in matters TB disease[15].

Health care providers should tailor as health communication perceived to enhance message relevance and should have the skill to identify situations[18]. It is due to a different channel to reach hard to reach the population in the different region.

\section{Conclusion}

Our findings have suggested areas of intersection between a patient's health care and their cultural-religious beliefs that need a cultural and religious approach to assist in increase TB 
knowledge and Awareness for TB Program. Reaching hard to reach population through religious, informal leaders and traditional music can increase TB knowledge and Awareness for TB Program in West Sumatera Province, Indonesia.

\section{References}

[1] Organization WH. Global Tuberculosis Report 2015. 20th Ed ed. Geneva: World Health Organization; 2015. $192 \mathrm{p}$.

[2] Health IMo. National Tuberculosis Program: Annual Report 2016: Indonesia Ministry of Health; 2015.

[3] Abebe G, Deribew A, Apers L, Woldemichael K, Shiffa J, Tesfaye M, et al. Knowledge, health seeking behavior and perceived stigma towards tuberculosis among tuberculosis suspects in a rural community in southwest Ethiopia. PLoS One. 2010;5(10):e13339.

[4] Khan JA, Irfan M, Zaki A, Beg M, Hussain SF, Rizvi N. Knowledge, attitude and misconceptions regarding tuberculosis in Pakistani patients. Journal of Pakistan Medical Association. 2006;56(5):211.

[5] Enwuru C, Idigbe E, Ezeobi N, Otegbeye A. Care-seeking behavioural patterns, awareness and diagnostic processes in patients with smear-and culture-positive pulmonary tuberculosis in Lagos, Nigeria. Transactions of the Royal Society of Tropical Medicine and Hygiene. 2002;96(6):614-6.

[6] Jit M, Stagg HR, Aldridge RW, White PJ, Abubakar I. Dedicated outreach service for hard to reach patients with tuberculosis in London: observational study and economic evaluation. 2011.

[7] Maher D. The role of the community in the control of tuberculosis. Tuberculosis. 2003;83(1):177-82.

[8] Poudel KC, Jimba M, Poudel-Tandukar K, Wakai S. Reaching hard-to-reach migrants by letters: An HIV/AIDS awareness programme in Nepal. Health \& place. 2007;13(1):173-8.

[9] Sengupta S, Pungrassami P, Balthip Q, Strauss R, Kasetjaroen Y, Chongsuvivatwong V, et al. Social impact of tuberculosis in southern Thailand: views from patients, care providers and the community. The International Journal of Tuberculosis and Lung Disease. 2006;10(9):1008-12.

[10] Simmons D, Voyle JA. Reaching hard-to-reach, high-risk populations: piloting a health promotion and diabetes disease prevention programme on an urban marae in New Zealand. Health Promotion International. 2003;18(1):41-50.

[11] Minetti A, Hurtado N, Grais RF, Ferrari M. Reaching hard-to-reach individuals: nonselective versus targeted outbreak response vaccination for measles. American journal of epidemiology. 2014;179(2):245-51.

[12] DeHaven MJ, Hunter IB, Wilder L, Walton JW, Berry J. Health programs in faith-based organizations: are they effective? American journal of public health. 2004;94(6):1030-6.

[13] Trinitapoli J, Ellison CG, Boardman JD. US religious congregations and the sponsorship of health-related programs. Social Science \& Medicine. 2009;68(12):2231-9.

[14] [Toni-Uebari TK, Inusa BP. The role of religious leaders and faith organisations in haemoglobinopathies: a review. BMC blood disorders. 2009;9:6.

[15] Pirkani GS, Qadeer E, Ahmad N, Razia F, Khurshid Z, Khalil L, et al. Impact of training of religious leaders about tuberculosis on case detection rate in Balochistan, Pakistan. J Pak Med Assoc. 2009;59(Suppl 1):s114-s7.

[16] Green EC. The impact of religious organizations in promoting HIV/AIDS prevention. Challenges for the church: AIDS, malaria \& TB. 2001.

[17] Houston HR, Harada N, Makinodan T. Development of a culturally sensitive educational intervention program to reduce the high incidence of tuberculosis among foreign-born Vietnamese. Ethnicity and Health. 2002;7(4):255-65.

[18] Dutta-Bergman MJ. Theory and Practice in Health Communication Campaigns: A Critical Interrogation. Health Communication. 2005;18(2):103-22. 